\title{
Coherence in Disordered Emitters Coupled to Surface and Long-Range Plasmons
}

\author{
J.M. Benoit, K. Chevrier, C. Symonds, J. Paparone, and J. Bellessa \\ Institut Lumière Matière, Université de Lyon, Université Claude Bernard Lyon 1-CNRS, \\ F-69622, Villeurbanne, France
}

\section{EXTENDED ABSTRACT}

Localized and delocalized plasmons in metallic nanostructures are associated with a strongly confined electromagnetic field, inducing an enhanced interaction with emitters located in the close environment of the metal. When the plasmon/emitter interaction overtakes the damping in the system, the system enters into strong coupling regime leading to light-matter hybridization [1]. This strong coupling has been observed with a large number of materials, in particular disordered materials which are constituted by a collection of independent emitters (molecules, semiconductor quantum dots...). The spatial and dynamic properties of an assembly of molecules in strong coupling are drastically modified compared to the same molecules in weak coupling [2].

We will present our on-going research on strong coupling between plasmons and molecular emitters. If we take into account the microscopic structure of the molecular film, collective effects between the delocalized plasmon and the set of molecules occurs. The excitations are not localized in a single emitter anymore but delocalized on a large number of molecules due to the formation of an extended hybridized state over several microns which modifies various properties of molecular materials like conductivity or reactivity [3]. However, the extension of the coherent state is limited by the reduced plasmon propagation length, due to metal losses. We will show that the coherence length can be extended using a low loss surface plasmon in a symmetrical structure leading to Long Range Surface Plasmon (LRSP) [4,5] (Fig. 1a). Simulations using a transfer matrix method are performed to optimize the parameters of the LRSP formation and its interaction with the aggregated dye and compared to experiments. After the demonstration of strong coupling between aggregated dyes and long range plasmon (Fig. 1b), we will evaluate the extension of the coherent polaritonic mode in the structures. A coherence length up to several tens of microns can be achieved. The parameters of these modes will be discussed to optimize the interaction with the organic material.
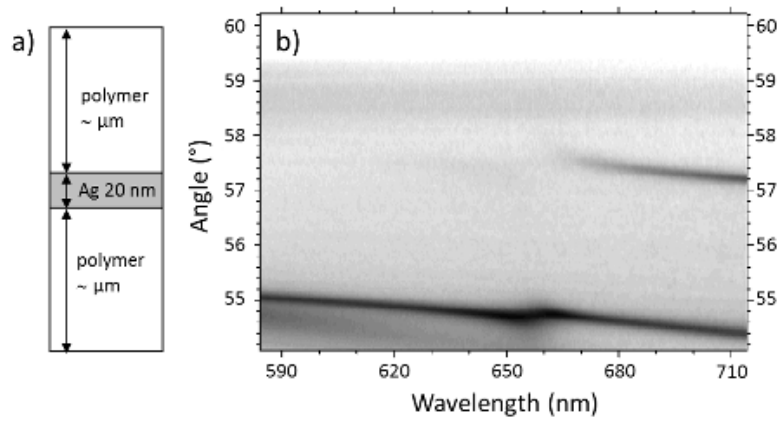

Figure 1: a) Schematic of the sample structure for surface plasmon long range generation; b) Experimental relation dispersion of plasmon strongly coupled with molecular emitters.

\section{REFERENCES}

[1] J. Bellessa, C. Bonnand, J.C. Plenet, and J. Mugnier: Strong coupling between surface plasmons and excitons in an organic semiconductor, Physical Review Letters 93, 036404 (2004).

[2] S. Aberra Guebrou, C. Symonds, E. Homeyer, J.C. Plenet, Y.N. Gartstein, V.M. Agranovich, and J. Bellessa: Coherent emission from a disordered semiconductor induced by strong coupling with surface plasmons, Physical Review Letters 108, 066401 (2012).

[3] E. Orgiu E. Orgiu, J. George,J. A. Hutchison, E. Devaux, J. F. Dayen, B. Doudin, F. Stellacci, C. Genet, J. Schachenmayer, C. Genes, G. Pupillo, P. Samorì, and T. W. Ebbesen: Conductivity in organic semiconductors hybridized with the vacuum field, Nature Mater. 14, 1123 (2015).

[4] P. Berini: Figures of merit for surface plasmon waveguides, Optics Express 14, 13030 (2006).

[5] D. Sarid: Long-range surface-plasma waves on very thin metal films, Phys. Rev. Lett. 47, 1927 (1981). 